\title{
Compensation for Harmonic Flux and Current of Permanent Magnet Synchronous Motor by Injected Harmonic Voltage
}

\author{
Jun Lu' ${ }^{1}$, Jianguo Yang ${ }^{1}$, Steven Y. Liang ${ }^{2,3}$, Ruirong Ren ${ }^{3}$ \\ ${ }^{1}$ School of Mechanical Engineering, Donghua University, Shanghai, 20060, China \\ ${ }^{2}$ School of Mechanical Engineering, Georgia Institute of Technology, Atlanta, GA 30332, United States \\ ${ }^{3}$ Donghua University, Shanghai, 20060, China
}

\begin{abstract}
High-order harmonic flux and current of permanent magnet synchronous motor (PMSM) are generated by the distortion of air-gap magnetic field and the nonlinear characteristics of inverter, resulting in the ripple of electromagnetic torque. In this paper, a novel harmonic compensation algorithm was proposed to solve this problem. Based on the harmonic model of the PMSM, harmonic voltage is injected to reduce harmonic components of the motor flux and current. By extraction of harmonic flux/current and injection of harmonic voltage in real-time, high-order harmonics of flux generated by the distortion of air-gap magnetic field and the nonlinear characteristics of inverter is counteracted. Thereby, distortion of motor current is reduced. The effectiveness of the algorithm is validated by simulation and experimentation.
\end{abstract}

Keywords-harmonic compensation; flux and current; PMSM; injected harmonic voltage.

\section{INTRODUCTION}

Stability of electromagnetic torque is important for motor control [1], influenced by high-order harmonic flux and current. Excitation magnet filed of rotor is not sinusoidal with odd harmonics in PMSM, especially in interior PMSM [2]. For suppression of harmonic components, improvement on structure design is researched for ideal excitation magnet filed using finite element analysis [3-8]. But it leads to limited effect and higher manufacturing cost. Other studies are focused on harmonic currents caused by nonlinear characteristic of switch device of inverter such as voltage drop and dead-time. Some methods are presented including non dead-time control [9], time compensation [10] and voltage compensation based on current feedback [11]. But effect is limited due to difficulty in current zero-cross detection, additional hardware, complicated algorithm and low adaptability [12].

Since harmonics are generated in different factors and vary with the motor operating point, the traditional harmonic compensation is very complex and its effect is not ideal. A novel harmonic compensation algorithm is proposed so that not only particular but also almost harmonic components are suppressed in the entire governing system. Effect of this method is validated through simulation and experimentation. In the experimental platform of vector control of a 400 watt PMSM, DSP-TMS28068M and Intelligent Power Module (IPM) is used as the controller and the inverter, respectively. The harmonic component and total harmonic distortion (THD) of motor current are compared and analyzed before and after using this method without load.

\section{PMSM HARMONIC MODEL}

In PMSM steady operation, the motor current is distorted and contains 5th, 7th, 11th, 13th harmonics because of air-gap magnetic field distortion, inverter voltage drop, dead time and other factors. The motor torque ripple and flux distortion are caused by these harmonics, especially5th and 7th harmonic by which 6th torque/flux harmonic is generated [13].

In static three phase coordinate, 5th harmonic voltage vector rotates at speed of $5 \omega$ in the reverse direction of fundamental voltage vector. 7 th harmonic voltage vector rotates at speed of $7 \omega$ in the same direction of fundamental voltage vector. 5th and 7th harmonics of PMSM three phase voltages are expressed in equation 1 :

$$
\left\{\begin{array}{l}
u_{a}=u_{1} \sin \left(\omega t+\theta_{1}\right)+u_{5} \sin \left(-5 \omega t+\theta_{2}\right)+u_{7} \sin \left(7 \omega t+\theta_{3}\right)+\ldots \\
u_{b}=u_{1} \sin \left(\omega t+\theta_{1}-\frac{2 \pi}{3}\right)+u_{5} \sin \left(-5 \omega t+\theta_{2}-\frac{2 \pi}{3}\right)+u_{7} \sin \left(7 \omega t+\theta_{3}-\frac{2 \pi}{3}\right)+\ldots \\
u_{c}=u_{1} \sin \left(\omega t+\theta_{1}+\frac{2 \pi}{3}\right)+u_{5} \sin \left(-5 \omega t+\theta_{2}+\frac{2 \pi}{3}\right)+u_{7} \sin \left(7 \omega t+\theta_{3}+\frac{2 \pi}{3}\right)+\ldots
\end{array}\right.
$$

According to the principle of coordinate transformation from a-b-c to d-q, the vector rotating at the same speed and in the same direction of $d-q$ synchronous rotating coordinate is a direct component. Thus, in $\mathrm{d}-\mathrm{q}$ coordinate of 5 th harmonic, 5th harmonic is a direct component while fundamental and other harmonics are all alternate components, as the same in 7th coordinate. Voltage and flux with harmonic components in $\mathrm{d}-\mathrm{q}$ coordinate of 5th harmonic is respectively shown in equation 2 and equation 3.

$$
\left\{\begin{aligned}
u_{d}^{*}= & -\omega L_{q} i_{q 1} \sin \left(6 \omega t+\theta_{4}\right)+R_{s} i_{d 1} \cos \left(6 \omega t+\theta_{4}\right)+5 \omega L_{q} i_{q 5 t h}+R_{s} i_{d 5 t h} \\
& -7 \omega L_{q} i_{7} \sin \left(12 \omega t+\theta_{6}\right)+R_{s} i_{7} \cos \left(12 \omega t+\theta_{6}\right)+\ldots \\
u_{q}^{*}= & \omega L_{d} i_{d 1} \sin \left(6 \omega t+\theta_{4}\right)+R_{s} i_{q 1} \cos \left(6 \omega t+\theta_{4}\right)+5 \omega L_{d} i_{d 5 t h}+R_{s} i_{95 t h} \\
& +7 \omega L_{d} i_{7} \sin \left(12 \omega t+\theta_{6}\right)+R_{s} i_{7} \cos \left(12 \omega t+\theta_{6}\right)+\ldots
\end{aligned}\right.
$$




$$
\left\{\begin{array}{l}
\psi_{d}^{*}=L_{d} i_{d}+\psi_{f}-\psi_{d 6} \cos (6 \omega t) \\
\psi_{q}^{*}=L_{q} i_{q}+\psi_{q 6} \sin (6 \omega t)
\end{array}\right.
$$

\section{HARMONIC REDUCTION ALGORITHM}

\section{A. Extraction model of harmonic flux}

Harmonic components are added into three phase reference voltages of PMSM so that flux/current harmonics are reduced. As shown in Figure 1, an algorithm is designed for harmonic flux/current extracted from actual current.

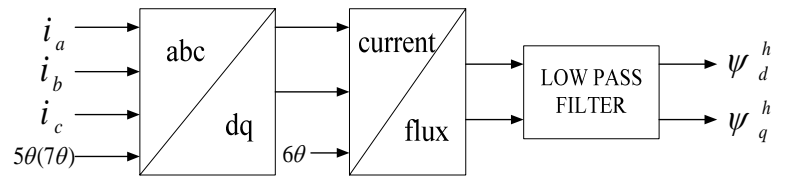

Figure 1. Extraction algorithm of 5th and 7th current harmonics

In corresponding d-q coordinates, 5th and 7th harmonics are respectively direct components while fundamental and other harmonics are all alternate components. Thus, these two harmonics can be extracted by low-pass filter.

\section{B. Compensation model of harmonic flux}

According to equation 4 and 6, Compensation model of harmonic flux using 5 th and 7th current harmonics is shown in Figure 2.

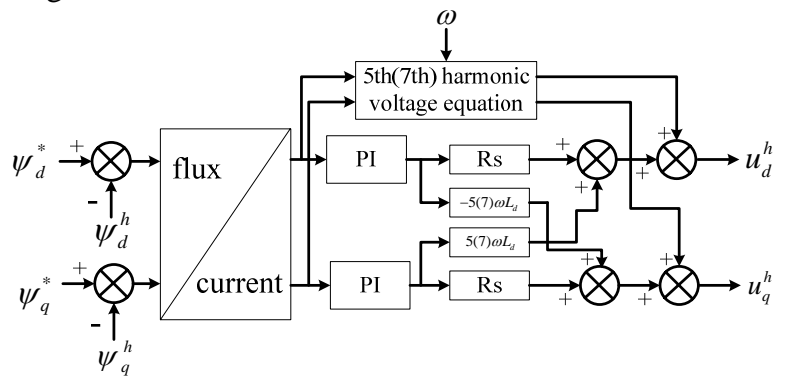

Figure 2. Reduction algorithm of 5th and 7th current harmonics

In PMSM steady-state harmonic voltage equations, $\mathrm{d}$-axis and q-axis voltages are respectively affected by q-axis and d-axis currents. According to these equations, a PI controller with cross-product term is designed so that accurate harmonic voltages can be obtained in any PMSM with different parameters and operating points.

\section{Coordinate transformation of harmonic voltage}

The harmonic voltages calculated by harmonic reduction algorithm are inversely transformed and accumulated. The compensation in static three phase coordinate is shown in Figure 3.

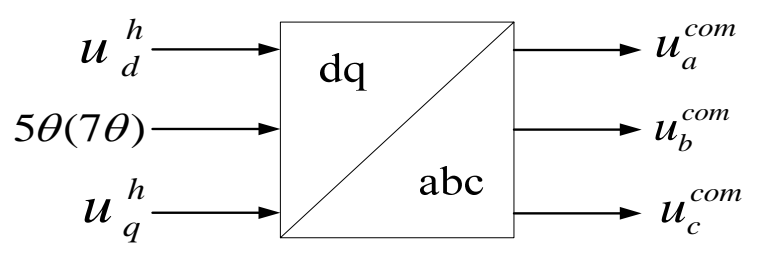

Figure 3. Transformation of harmonic voltages form d-q to a-b-c

\section{ANALYSIS OF SIMULATION AND EXPERIMENTATION}

\section{A. Analysis of simulation}

The simulation result of Matlab/Simulink is analyzed and compared so that effect of compensation of harmonic flux and current can be validated. In simulation, harmonic current is deliberately generated for validation through the setting of voltage drop and dead time of inverter. Since the motor model with distorted air-gap magnetic field is very complicated, the harmonic current caused by this factor is not considered. Parameters of PMSM are given in Table I.

TABLE I. PARAMETERS OF PMSM

\begin{tabular}{|c|c|c|c|}
\hline Parameter & Value & Parameter & Value \\
\hline $\begin{array}{c}\text { Load torque } \\
\mathrm{Tm} /(\mathrm{Nm})\end{array}$ & 0 & Rs/ohm & 2.657 \\
\hline $\mathrm{Ld} / \mathrm{mH}$ & 6.7 & $\mathrm{Lq} / \mathrm{mH}$ & 6.7 \\
\hline Inertia J/(Kgm2) & 0.006 & Pair of poles & 1 \\
\hline
\end{tabular}

In simulation, harmonic current is deliberately generated for validation through the setting of voltage drop and dead time of inverter. Parameters of inverter are given in Table II.

TABLE II. PARAMETERS OF INVERTER

\begin{tabular}{|c|c|c|c|}
\hline Parameter & value & Parameter & value \\
\hline Udc/V & 300 & Carrier frequency f/KHz & 11.7 \\
\hline Carrier period T/us & 85.5 & Dead time td/us & 4 \\
\hline IGBT on-time ton/us & 1 & IGBT off-time toff/us & 2 \\
\hline
\end{tabular}

As shown in Figure 4 and Figure 5, simulation results before and after using harmonic compensation algorithm are compared when PMSM operates in no-load, 180r/min and $3 \mathrm{~Hz}$ current fundamental frequency.

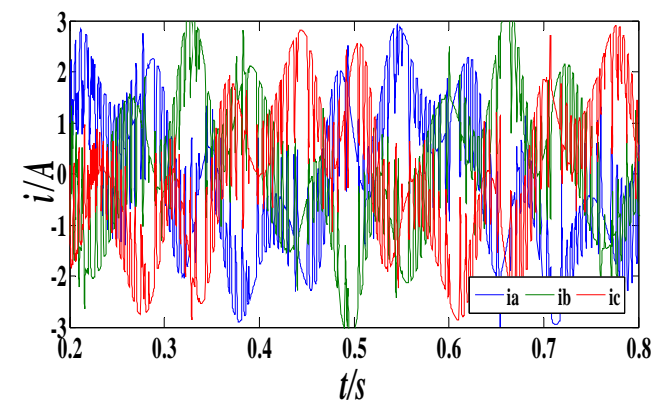

(a) 


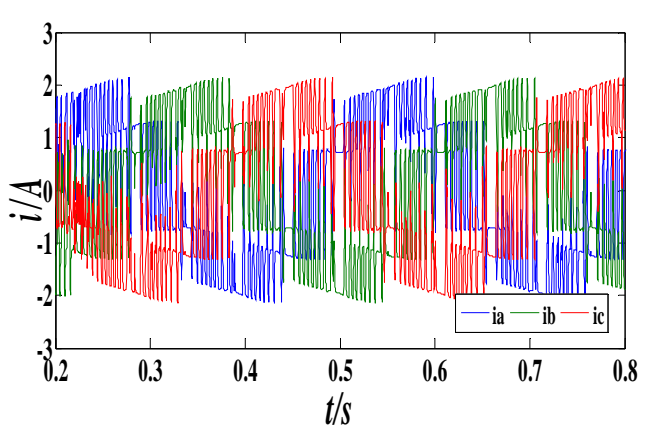

(b)

Figure 4. Phase current before and after algorithm

Current harmonic gets heavy and shows the wave seriously distorted without applying harmonic reduction algorithm as shown in Figure 4(a). After utilizing harmonic reduction algorithm, motor currents get obviously improved for standard sine as shown in Figure 4(b).

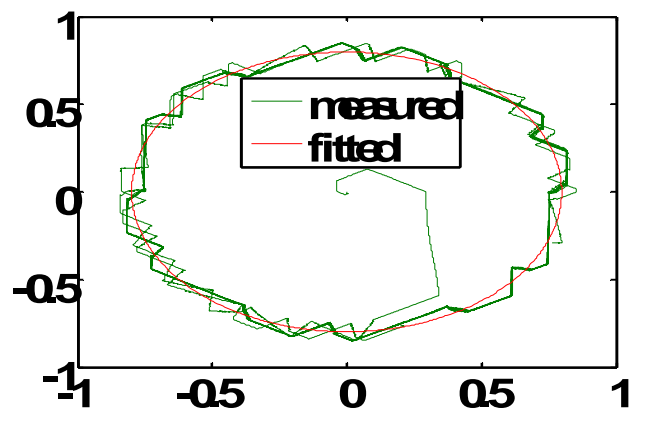

(a)

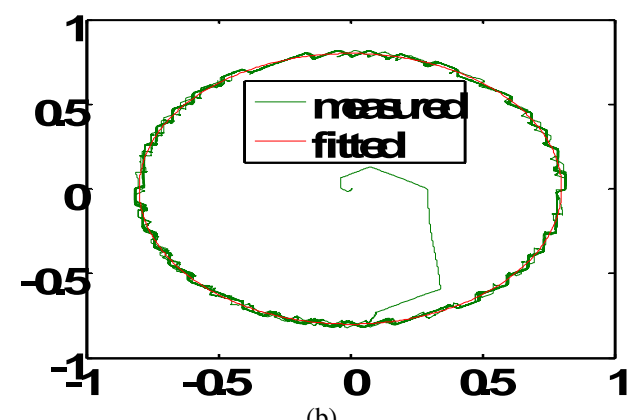

(b)

Figure 5. Flux trajectory before and after algorithm

In Figure 5, flux trajectory is reduced in distortion and fits to a standard circle. RMS is 0.0301 and 0.0124 before and after getting improved, respectively.

\section{B. Analysis of experimentation}

In the experimental platform of vector control of a 400 watt PMSM, DSP-TMS28068M and Intelligent Power Module (IPM) is used as the controller and the inverter respectively, as shown in Figure 6.

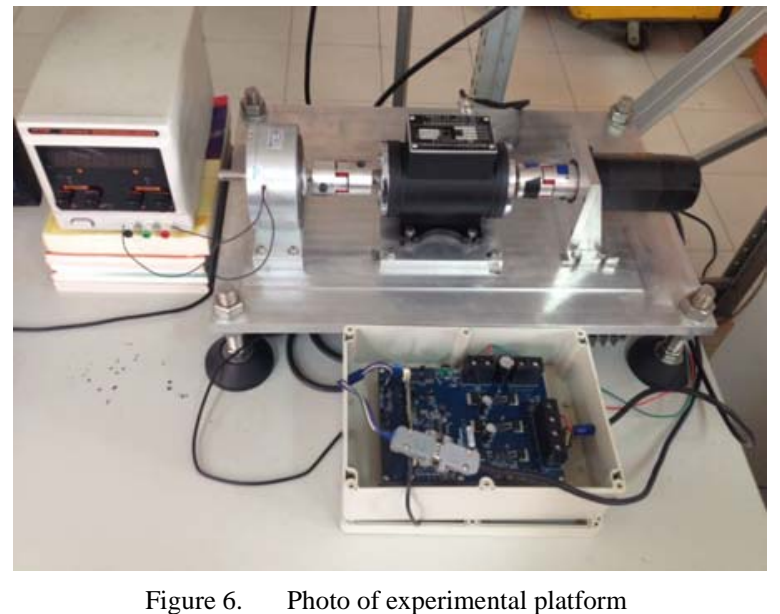

Rated parameter of PMSM is : $\mathrm{P}_{\mathrm{N}}=400 \mathrm{~W}, \mathrm{U}_{\mathrm{N}}=60 \mathrm{~V}$, $\mathrm{L}=0.2 \mathrm{mH}$, rotor flux $=0.08 \mathrm{~Wb}, \quad \mathrm{R}_{\mathrm{S}}=300 \mathrm{mOhm}$, $\mathrm{n}_{\mathrm{N}}=3000 \mathrm{r} / \mathrm{min}$, pair of poles is 1 . Parameter of inverter is: PWM carrier frequency is $2.95 \mathrm{kHz}$, dead time is $3.85 \mathrm{us}$, IGBT forward voltage drop is 2.3V, IGBT reverse voltage drop is $3.7 \mathrm{~V}$. As shown in Figure 7 and Figure 8, the harmonic component and THD of motor current before and after using this method are compared and analyzed when PMSM operates in no-load, $180 \mathrm{r} / \mathrm{min}$ and $3 \mathrm{~Hz}$ current fundamental frequency.

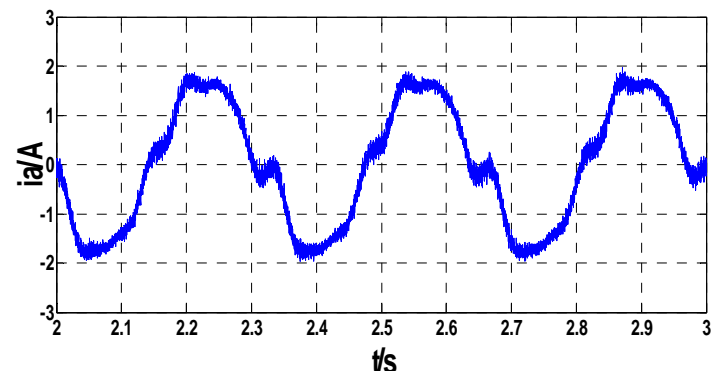

(a)

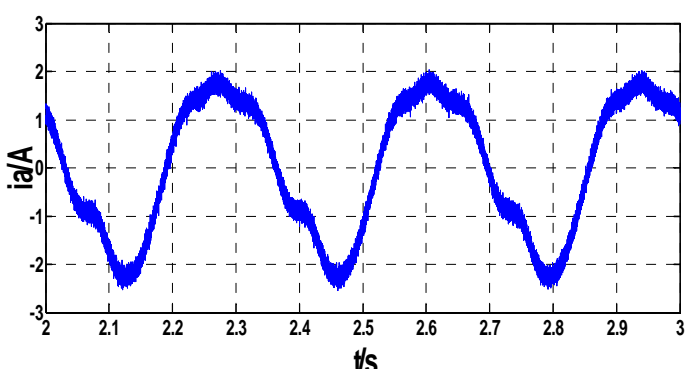

(b)

Figure 7. Phase current before and after algorithm

Current harmonic gets heavy and shows the wave seriously distorted without applying harmonic reduction algorithm as shown in Figure 7(a). After utilizing harmonic reduction algorithm, motor currents get obviously improved for standard sine as shown in Figure 7(b). 

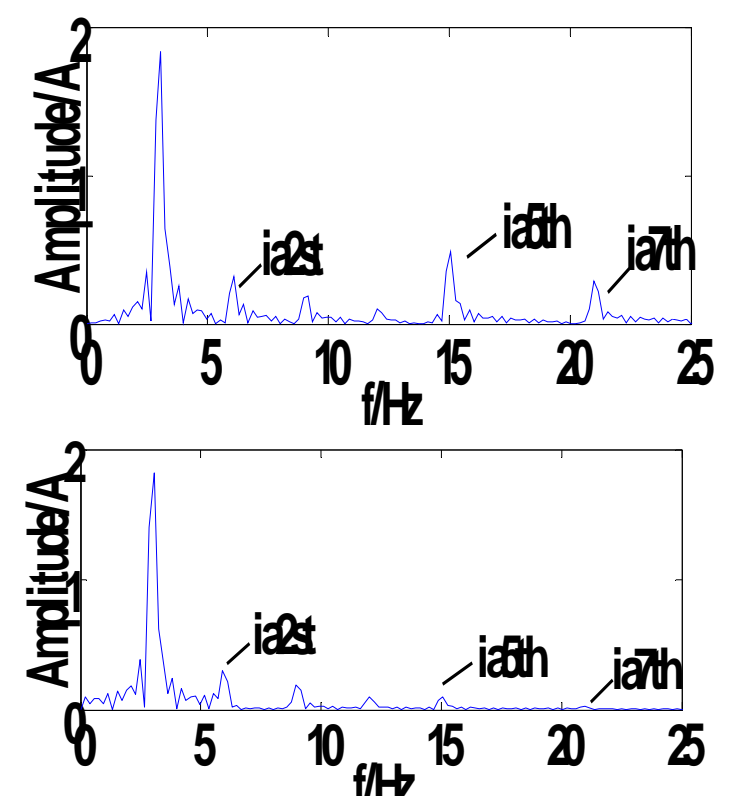

Figure 8. Frequency spectrum of phase current before and after algorithm

Figure 8 shows the amplitude of 5 th and 7 th current harmonics go obviously down in their corresponding $d-q$ synchronous rotating coordinate. 5th and 7th current harmonics drop from $18.60 \%$ to $2.91 \%$, from $6.98 \%$ to $1.67 \%$, respectively. Total harmonic distortion (THD) gets down from $26.54 \%$ to $5.39 \%$. 5th and 7 th harmonics are therefore suppressed with the use of harmonic compensation algorithm. Compared with simulation result, other high-order harmonics such as 2nd harmonic appear in addition to 5th and 7th harmonics as shown in figure 8. It results from the distortion of air-gap magnetic field and the nonlinear characteristics of inverter that can't be included in simulation model. Therefore, reduction of 5 th and 7 th current harmonics caused by these factors is validated.

However, 5th and 7th current harmonics are not kept to zero strictly both in simulation and in experimentation since real-time and accurate rotor position is needed for harmonic reduction algorithm. Method for decreasing its influence to the effect of harmonic reduction algorithm will be presented for further research.

\section{CONCLUSIONS}

In this paper, a novel harmonic compensation algorithm was proposed to achieve a stable running of PMSM without torque ripple caused by flux/current harmonics. Simulation and experimentation have proved that flux/current harmonics existed in governing system are reduced. Mathematic models of 5th and 7th harmonics deduced in this paper can be used for accurate analysis of voltage and current harmonics in governing system. To reduce the harmonics generated by IGBT dead time and voltage drop, harmonic compensation is used without complication of zero-crossing current detection in traditional method. It is also validated that the harmonics caused by distortion of air-gap magnetic field are suppressed.

\section{REFERENCES}

[1] Wu Maogang, Zhao Rongxiang, Tang Xinzhou, Study of vector-controlled permanent magnet synchronous motor at low speed and light load. Transactions of China Electrotechnical Society, 20(7), pp. 87-92, 2005.

[2] Li Jingcan, Liao Yong, Model of permanent magnet synchronous motor considering saturation and rotor flux harmonics. Proceedings of the CSEE, 31(3), pp. 60, 2011.

[3] K J Tseng,S B Wee, Analysis of Flux Distribution and Core Losses in Interior Permanent Magnet Motor. IEEE Transactions on Energy Conversion, 14(4), pp. 969, 1999.

[4] Rich Schiferl, Core Loss in Buried Magnet Permanent Magnet Synchronous Motors. IEEE Trans on Energy Conversion, 4(2), pp. 279, 1989.

[5] Han S-H, Jahns T M, Zhu Z Q, Analysis of rotor core eddy-current losses in interior permanent-magnet synchronous machines. IEEE Transactions on Industry Applications, 46(1), pp. 196, 2010.

[6] Xiao $\mathrm{X}$, Chen $\mathrm{C} \mathrm{M}$, Reduction of torque ripple due to demagnetization in PMSM using current compensation. IEEE Transactions on Applied Superconductivity, 20(3), pp. 1068, 2010.

[7] Lee G-H, Kim S-I, Hong J-P, et al, Torque ripple reduction of interior permanent magnet synchronous motor using harmonic injected current. IEEE Transactions on Magnetics, 44(6), pp. 1582, 2008.

[8] Seok-Hee Han, T. M Jahns, W. L. Soong, Torque ripple reduction in interior permanent magnet synchronous machines using the principle of mutual harmonics exclusion. IEEE Industry Applications Conference, pp. 558-565, 2007

[9] Lin Y K, Lai Y S, Dead-time elimination of PWM-controlled inverter converter without separate power sources for current polarity detection circuit. IEEE Transactions on Industrial Electronics, 56(6), pp. 2121-2127, 2009.

[10] Kerkman R J, Leggate D, Schlegel D W, et al, Effects of parasitics on the control of voltage source inverter. IEEE Transactions on Power Electronics, 18(1), pp. 140-150, 2003.

[11] Urasaki N, Senjyu $T$, Uezato $K$, et al, An adaptive dead-time compensation strategy for voltage source inverter-fed motor drives. IEEE Transactions on Power Electronics, 20(5), pp. 1150-1160, 2005.

[12] Kim S Y, Lee W, Rho M S, et al, Effective dead-time compensation using a simple vectorial disturbance estimator in PMSM drives. IEEE Transactions on Industrial Electronics, 57(5), pp. 1609-1614, 2010.

[13] Yong Liao, Dawei Xiang, Li Ran, et al, Analysis of harmonic transfer in an AC excited generator including speed ripple. IEEE 28th Annual Conference of the Industrial Electronics Society, pp. 1162-1166, 2002. 